\begin{tabular}{|l|l|l|}
\hline & PPM $(\mathbf{n}=35)$ & CRT $(\mathbf{n = 2 1})$ \\
\hline Age (years, mean \pm SD) & $75.9 \pm 13.6$ & $67.3 \pm 11.3$ \\
\hline Male & $8(22.9 \%)$ & $n=13(61.9 \%)$ \\
\hline Systolic Function (LVEF \%) & $56 \pm 4.3$ & $53.2 \pm 2.8$ \\
\hline Ablation Procedure Time (mins) & $55.0 \pm 28.89$ & $50.7 \pm 21.98$ \\
\hline Device Procedure Time (mins) & $85.8 \pm 34.77$ & $132.1 \pm 40.94$ \\
\hline Ischaemic Heart Disease & $1(2.9 \%)$ & $3(14.2 \%)$ \\
\hline Alive & $31(88.6 \%)$ & $20(95.2 \%)$ \\
\hline Follow Up (days, mean \pm SD ) & $370 \pm 191$ & $319 \pm 208$ \\
\hline
\end{tabular}

prophylactic CRT implantation in patients with preserved LV was addressed in the BIOPACE trial, however these results were not formally published and controversy still exists about the type of device to implant in patients with near normal LV function. ${ }^{3}$

Our study describes the variability in our clinical practice given the split in PPM vs CRT in patients with near normal LV function. The increased age and mortality trend in the PPM group may reflect an increased frailty or co-morbidity compared with the CRT group. However, long term clinical outcomes are required in order to provide clarity on this important clinical decision.

Conflict of Interest None

\section{DO PATIENTS NOTICE A DIFFERENCE BETWEEN DIFFERENT TYPES OF IMPLANTABLE LOOP RECORDERS?}

${ }^{1}$ Natasha Bloodworth* ${ }^{*}{ }^{2}$ Sophia John, ${ }^{1}$ Mark Earley, ${ }^{2}$ Anthony Chow, ${ }^{2}$ Martin Lowe, ${ }^{2}$ Jonathan M Behar. ${ }^{1}$ Barts Health NHS Trust; ${ }^{2}$ Barts Heart Centre

\subsection{6/heartjnl-2019-BCS.46}

Introduction Indications for implantable loop recorder (ILR) implantation include unexplained syncope or pre-syncope, palpitations and cryptogenic stroke. Referral for ILRs continues to increase and the new generation of injectable devices lend themselves to less invasive implantation in the outpatient setting rather than the catheter laboratory. Our centre is currently implanting the Linq (Medtronic) and Confirm (Abbott) devices. The latter is a recently introduced product and we aimed to compare the two devices at our centre using patient reported outcomes.

Methods This was a single centre retrospective analysis of patients who attended Barts Heart Centre for an outpatient ILR implant in February-November 2018 when both Linq and Confirm devices were being used. A questionnaire was designed to assess the patient experience at all stages of ILR implant and follow up. Questions 1 and 2 were designed to gauge patient's opinions on the quality of information provided before implantation; questions 3,4 and 7 were used to find out about the patient's experience at the implant visit and questions 5, 6, 8 and 9 asked about the patient's experience following device implantation. All questions were rated from 1 to 5 on a Likert scale with 5 being the best possible score. Questionnaires were administered over the phone. We compared the scores using unpaired t-tests.

Results A total of 38 respondents were included in the analysis (Linq and Confirm $\mathrm{n}=19$ each). All patients were implanted in the outpatient setting by a trained nurse along with a cardiac physiologist. Average age did not differ significantly between groups (Linq $61 \pm 18$ vs. Confirm $52 \pm 19$ years, $\mathrm{p}=0.01)$ and the most common implant indication was syncope/presyncope.

Overall Linqs scored significantly better than Confirms in terms of the patient experience for implant and follow up (Linq $40 \pm 3.77$ vs. Confirms $36 \pm 6.07 ; p=0.01$ ). Patients scored Linqs significantly higher for healing following device implantation when compared to Confirms (Linqs $4.73 \pm 0.45$ vs. Confirms $3.73 \pm 1.32, p=0.01$ ) (See figure 1 ). There were also trends towards less perceived pain during device implant for those implanted with Linq verses those receiving Confirms (Linqs $4.63 \pm 0.59$ vs. Confirms 4.16 $\pm 0.89, \quad \mathrm{p}=0.06$ ) and patient's with Linqs had greater confidence in their devices ability to successfully diagnose their symptoms (Linqs $4.21 \pm 0.60$ vs. Confirms $3.58 \pm 1.21, p=0.05$ ). The two manufacturers devices scored similarly for quality of information provided pre-implant (Linq 4.26 \pm 1.19 vs. Confirm 3.95 \pm 0.97 , $\mathrm{p}=0.38$ ), how comfortable the device is to live with (Linq $4.32 \pm 0.89$ vs. Confirm $3.79 \pm 1.27, \mathrm{p}=0.15)$ and ease of use of home monitoring equipment (Linq $4.42 \pm 0.69$ vs. Confirm $3.79 \pm 1.51, \mathrm{p}=0.11)$. Qualitatively, older patients reported needing more help to use the Confirm smart phone application based home monitor, whereas younger patient's found system very easy to use.

Discussion/Conclusions Patients report good experiences when having ILRs implanted in the outpatient setting. From this limited cohort, patients report a better overall experience with the Linq than the Confirm device. However, it should be noted that the Confirm device is a new product and medical staff exposure and experience with this is far less than with the Linq device; this may have influenced the results. Larger and randomised studies are needed to qualify and quantify any differences between these products.

Conflict of Interest None 